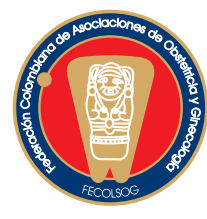

EDitorial

\title{
EL INSTITUTO MATERNO INFANTIL Y LOS PLANES DE REDUCCIÓN DE LA MORTALIDAD MATERNA: UN RETO PARA EL SISTEMA DE SEGURIDAD SOCIAL EN SALUD
}

Hernando Gaitán, M.D., MSc.*

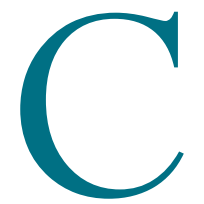

ada minuto muere una mujer por complicaciones del embarazo o el parto en el mundo. Por cada mujer que muere, hay 30 más que presentan secuelas de las complicaciones de este periodo, ${ }^{1}$ sin mencionar el serio impacto que se genera sobre la salud de los recién nacidos. Los hijos de madres que fallecen por problemas relacionados con el embarazo, parto o puerperio tienen 10 veces más riesgo de morir que los recién nacidos de madres que sobreviven. ${ }^{2}$ La mortalidad materna es un indicador del desarrollo de una nación, es una señal acerca de la disparidad e inequidad en la distribución de la riqueza entre países y dentro de ellos mismos, entre hombres y mujeres, así como también brinda una seña del sitio que ocupa la mujer en una sociedad. ${ }^{3}$

En Colombia, la razón de mortalidad materna había mostrado una disminución en la última década. Para Bogotá en el año 2000, la Secretaría de Salud informó una razón de mortalidad materna de 77 x 100.000 nacidos vivos, mientras el DANE registró para el mismo año una razón de 86 x 100.000 nacidos vivos. Sin embargo, se observa una tendencia, sino a incrementar, sí por lo menos a permanecer en una situación estable, con una media de aproximadamente 7 muertes semanales entre el 2001 y el 2004. ${ }^{4}$

Esta situación, si bien podría obedecer a un mejor registro de la mortalidad materna, es posible que también sea resultado del estancamiento o deterioro de la atención materno perinatal en el país.

* Editor Revista Colombiana de Obstetricia y Ginecología. Profesor Titular. Universidad Nacional de Colombia.
La mortalidad materna obedece a razones como la pobreza, el bajo estatus de la mujer, escaso acceso a los servicios de salud, mala nutrición, problemas de transporte de las pacientes y servicios inadecuados, que explican que esta cifra sea en nuestro país hasta 20 veces más frecuente que en los países industrializados. Esta menor mortalidad no obedecería a que se presenten menos complicaciones en las pacientes de los países desarrollados, sino más bien a un acceso más rápido a servicios de atención de urgencias mejor calificados.

Se ha creado un modelo analítico para reducir la mortalidad materna que permite planear intervenciones en diferentes momentos. Este modelo se ha denominado "de las tres demoras". La primera es la que se presenta cuando la mujer no reconoce tempranamente los signos que indican que debe acudir al médico, o a pesar de reconocer que algo anda mal, demora en buscar la atención una vez se ha presentado una complicación. Una segunda tardanza es la causada por la dificultad para lograr el acceso a los servicios y la tercera cuando recibe de manera no oportuna las intervenciones destinadas a tratar la complicación en las instituciones de salud. En las dos primeras demoras actuarían las intervenciones culturales, económicas, sociales y administrativas. Sobre la última pretende actuar las intervenciones destinadas a mejorar la atención de la emergencia obstétrica. ${ }^{5}$

La acciones propuestas por el actual gobierno en el plan de choque contra la mortalidad materna, intentan entre otros aspectos que las gestantes acudan más temprano al control prenatal, empoderar 
al compañero en el cuidado de la mujer gestante y del recién nacido y lograr que la comunidad haga una discriminación positiva de la mujer en este momento de la vida. Por otra parte intentan coordinar las acciones entre los diferentes niveles para lograr un acceso rápido según el riesgo y buscar los responsables de los problemas de atención que conducen a la muerte materna. ${ }^{6}$ Como meta para el 2015, el gobierno nacional se ha comprometido a lograr que la mortalidad materna llegue al 45 x 100.000 recién nacidos vivos por medio del incremento en la cobertura de la seguridad social en el país, garantizar el acceso al control prenatal, atención de parto y puerperio de toda la población asegurada y no asegurada, fortalecimiento de la calidad de los servicios disponibles en las diferentes regiones del país, desarrollo de la vigilancia epidemiológica y registro de estadísticas vitales y uso de anticonceptivos. ${ }^{7}$ Las acciones están principalmente encaminadas a la detección temprana y calificación del riesgo.

Sin embargo, hay que tener en cuenta que el diagnóstico temprano no tiene el mismo efecto sobre la complicación materna que sobre la mortalidad perinatal, donde sí ejerce un positivo impacto. La urgencia obstétrica no es prevenible en muchos casos, a pesar de la detección y el manejo temprano de la patología de base. Por otra parte, los sistemas de clasificación de riesgo en la gestante no han dado los resultados esperados ya que no se puede predecir quién va a sufrir las complicaciones severas del embarazo. La clasificación de bajo y alto riesgo ha mostrado poca sensibilidad para predecir las complicaciones, debido a que un alto porcentaje de ellas se presentan en pacientes clasificadas con riesgo bajo. ${ }^{5}$

Se considera que entre el 1 y el $6 \%$ de los de los embarazos presentarán complicaciones médicas serias. ${ }^{89}$ Para la atención de estos eventos es clave contar con servicios de atención de urgencias obstétricas debidamente dotados y con el personal capacitado para su adecuada atención. Estos centros comprenden los llamados Centros de Cuidado de Atención de la Urgencia (Emergency Obstetric Care-EmOC).
Se ha descrito que entre 66 a $89 \%$ de las muertes maternas institucionales son evitables. Al revisar las causas de mortalidad materna en Colombia se encuentra que casi la mitad de las muertes son causadas por la hipertensión asociada al embarazo y sus complicaciones. Le siguen la hemorragia postparto, las alteraciones del parto y el trabajo de parto, las complicaciones del aborto y las infecciones posparto. En todas ellas se enfrenta el real momento de verdad en la atención de la emergencia obstétrica, que hace la diferencia entre la vida y la muerte.

En conclusión, por las siguientes razones, parece muy poco probable que el gobierno pueda cumplir estas metas con la estrategia propuesta:

- En Colombia el acuerdo 009-94 centró la atención materna infantil en entidades de primer nivel, donde muchas de instituciones no cuentan con la estructura adecuada ni el personal con la experticia necesaria para atender las complicaciones severas del embarazo, parto y puerperio que se presentan en pacientes clasificadas "de bajo riesgo".

- Las acciones de intervención centradas sobre los factores psicosociales y económicos tendrán un efecto más estable a mediano y largo plazo, a diferencia de las acciones destinadas al manejo óptimo y oportuno de la urgencia obstétrica, con repercusiones en el corto plazo.

- Para lograr un impacto a corto plazo, se requiere una mayor captación de la gestante por parte de los servicios de salud, mejorar el sistema de referencia y contra-referencia para que funcionen de nuevo las redes de atención materna y perinatal entre los hospitales de baja, media y alta complejidad y especialmente contar con servicios adecuadamente equipados para la atención de la emergencia obstétrica. Esta última estrategia no es solamente la de más rápida implementación con alto impacto, sino también la más costo-efectiva. ${ }^{10}$ De esta manera, se actuará sobre el 6 al 13\% de las pacientes obstétricas que tendrán complicaciones serias en el momento del parto y que representan la mayor carga sobre la mortalidad materna. 
Este aspecto no es priorizado en el Plan de Choque contra la Mortalidad Materna como tampoco en el Plan de Desarrollo al 2015. Lo que se observa en la práctica, más allá de la importante inversión en publicidad, ${ }^{7}$ es el cierre progresivo de los hospitales altamente especializados en atención materna y perinatal como el Hospital Infantil y el Instituto Materno Infantil. En este último caso se cerrarán los únicos servicios especializados en atención de preclampsia, infección obstétrica y hemorragia posparto, tres de las principales causas de mortalidad materna en el país. Esto tendrá repercusiones sobre los indicadores de salud materna e infantil a nivel de Bogotá y en la educación e investigación médica de todo el país.

Parte de la solución para salvar al Instituto Materno Infantil es transformarlo en un Instituto de Perinatología que sea el centro de cuidados para las pacientes con preeclampsia, infección obstétrica, y aquellas pacientes gestantes que requieran cuidados intensivos en el Distrito Capital y la región centrooriental del país. Allí se podrá centralizar el manejo de las pacientes con patologías especiales asociadas al embarazo como el parto pretérmino en edades gestacionales tempranas y la mola hidatidiforme.

Por otra parte, se podrán ofrecer servicios de atención ambulatoria en infecciones de transmisión sexual, endocrinología ginecológica y servicios de planificación a los grupos de desplazados, alternativas inexistentes en la actualidad, cumpliendo con la misión histórica de la Institución de atender a los más necesitados. Además servirá de centro de enseñanza en la atención de las patologías mencionadas, así como también para la educación médica continuada de los profesionales de los hospitales de la Secretaría Distrital de Salud. Por último, podrá continuar siendo el mayor centro de investigaciones en Colombia en las entidades que causan la mayor parte de las muertes materna y perinatal, con capacidad de captar recursos locales e internacionales.

Para reducir la mortalidad materna en Bogotá y en el país, se deben definir patologías prioritarias de atención materna perinatal de acuerdo a indicadores de impacto en la política de Salud Sexual y Reproduc- tiva, fortalecer la vigilancia epidemiológica, y la Red Materno-Perinatal a nivel local y regional. Se requiere incluir y fortalecer el IMI como Hospital de cuarto nivel en la red. Definir mecanismos administrativos que garanticen la remisión inmediata y atención prioritaria de las patologías antes mencionadas a los hospitales de tercero y cuarto nivel, aplicar el método de mejoramiento continuo de calidad en el área de atención materno perinatal y garantizar la atención universal de la gestante y el recién nacido.

\section{REFERENCIAS}

1. Maternal Mortality in 2000 - Estimates Developed by WHO, UNICEF and UNFPA. UNICEF Statistics: tomado de http://www.childinfo.org/eddb/mat_mortal/ el 20 de Junio de 2005.

2. Stopping the invisible epidemic of maternal deaths. World Health Organization. En: http://www.who.int/mediacentre/ news/releases/2004/pr65/en/. Tomado el 30 de septiembre de 2004.

3. Talking points. Maternal mortality. The Lancet 2004;363.

4. Boletín No 15 de mortalidad de notificación obligatoria semanal. Boletín Epidemiológico Semanal SIVIGILA Instituto nacional de Salud. Ministerio de la Protección Social , 29 de febrero - marzo 6 de 1004. Tomado de: http://www.col.opsoms.org/sivigila/2004/bole09_04\%20.htm

5. Maine D, Akalin MZ, Ward VM, Kamara A. The Design and Evaluation of Maternal Mortality Programs. Center for Population and Family Health, Columbia University, New York. 1997.

6. Plan de choque contra la mortalidad materna. Minutero de la Protección social 2003 en : http://www.minproteccionsocial. gov.co/MseContent/images/news/DocNewsNo513201.doc

7. Metas y estrategias de Colombia para el logro de los objetivos de desarrollo del Milenio - 2015: Consejo nacional de Politica Económica y Social CONPES y Departamento Nacional de Planeación, 2005 tomado de http://www.pnud.org.co/noticias/conpes\%20091.pdf

8. Waterstone M, Bewley S, Wolfe C. Incidence and predictors of severe obstetric morbidity: case-control study. BMJ 2001;322:1089-93.

9. Prual A, Bouvier-Colle MH, de Bernis L, Breart G. Severe maternal morbidity from direct obstetric causes in West Africa: incidence and case fatality rates. Bull World Health Organ 2000;78:593-602.

10. McCord C, Chowdhury Q. A cost effective small hospital in Bangladesh: what it can mean for emergency obstetric care. Int J Gynaecol Obstet. $2003 ; 81: 83-92$ 